\title{
Fontan tunnel puncture with 3-dimensional image fusion guidance for ablation of supraventricular arrhythmia in a patient with unique anatomy
}

\author{
Michał Gałeczka', Oskar Kowalski², Roland Fiszer ${ }^{1}$
}

'Department of Congenital Heart Defects and Pediatric Cardiology, FMS in Zabrze, Medical University of Silesia, Silesian Centre for Heart Diseases in Zabrze, Poland 2Department of Cardiology, Congenital Heart Diseases and Electrotherapy, FMS in Zabrze, Medical University of Silesia, Silesian Centre for Heart Diseases in Zabrze, Poland

\author{
Correspondence to: \\ Michał Gałeczka, MD \\ Department of Congenital \\ Heart Defects and Pediatric \\ Cardiology, FMS in Zabrze, \\ the Medical University of \\ Silesia in Katowice, Silesian \\ Center for Heart Diseases, \\ Curie-Skłodowskiej 9, \\ 41-800 Zabrze, Poland, \\ phone: +48322 7134 01, \\ e-mail: \\ michalgaleczka@gmail.com; \\ m.galeczka@sccs.pl \\ Copyright by the \\ Author(s), 2021 \\ Kardiol Pol. 2021: \\ 79 (7-8): 873-874 \\ DOI: 10.33963/KP.15971 \\ Received: \\ March 31, 2021 \\ Revision accepted: \\ April 16, 2021 \\ Published online: \\ April 26, 2021
}

A 29-year-old woman with single ventricle anatomy, namely dextroversion, pulmonary atresia, immense ventricular septal defect, and after Fontan pathway completion, was readmitted due to recurring and drug-resistant atrial tachyarrhythmia (AT).

The total cavopulmonary connection by lateral intra-atrial tunnel was performed at the age of 8 years with the use of a Gore-Tex patch. Afterward, she needed transcatheter closure of two shunts between the tunnel and right atrium (with coil and atrial septal occluder) and stent implantation to the left pulmonary artery. At the age of 27 years she underwent intra-tunnel radiofrequency ablation, however AT reappeared soon after. Due to gradual clinical deteriotation, she was qualified for ablation in a pulmonary venous atrium via tunnel puncture.

On admission, she presented in New York Heart Association II class, with oxygen saturation of $80 \%$ and hepatomegaly. Computed tomography (CT) was performed to outline the complex anatomy. A three-dimensional (3D) CT-derived model of a septum between the tunnel and the right atrium was prepared with image fusion software (VesselNavigator, Philips Healthcare) to serve as a roadmap overlay on a live X-ray image (Figure 1). The septum, including the previously implanted devices, was delineated, orientated in the thoracic cavity, the fluoroscopy projections planned, and the desired puncture location marked.

The procedure was performed under local anesthesia. Both femoral veins and the right jugular vein were accessed under ultrasonography guidance, and heparin was administered. 3D model was overlaid on the fluoroscopy image. Due to difficulty in stabilizing the long
7 French transseptal sheath with the Brockenbrough needle at the desired puncture location, a $15 \mathrm{~mm}$ lasso was inserted from the left femoral access to catch and fix the tip of the sheath (dilator). The septum was successfully punctured at the lowest possible level, slightly below the planned location. High-density mapping of both atria was conducted with the use of Ensite Precision Abbott 3D System, using Advisor HD grid mapping catheter. A diagnostic catheter was placed in the tunnel for reference. A slow-conduction area was found in the upper area of the left atrium's posterior wall, adjacent to the left superior pulmonary vein ostium. This area was responsible for arrhythmia of type figure 8 occurrence. An application was performed obtaining arrhythmia termination and inability to further inducement in programmed stimulation. Procedure and fluoroscopy times were 280 and 121 minutes, respectively, and the radiation dose was $121.3 \mathrm{Gycm}^{2}$.

During the 4-month observation, the AT episodes recurred, and flecainide was introduced.

The development of tachyarrhythmia is an important predictor of Fontan-type circulation failure [1]. Patients with lateral intra-atrial tunnel are prone to late-onset AT, which in the majority can be ablated from the tunnel [2-4]. The AT located in the pulmonary venous atrium is less likely to arise from surgical scars and may result from atrial fibrosis due to volume or pressure overload [2]. Those rare situations require a baffle puncture and cause substantial difficulty in atrial mapping.

Novel image fusion software suites allow for using preregistered CT or magnetic resonance imaging 3D datasets for live guidance of transcatheter interventions [5]. Such a tool has remarkable potential and can be particu- 


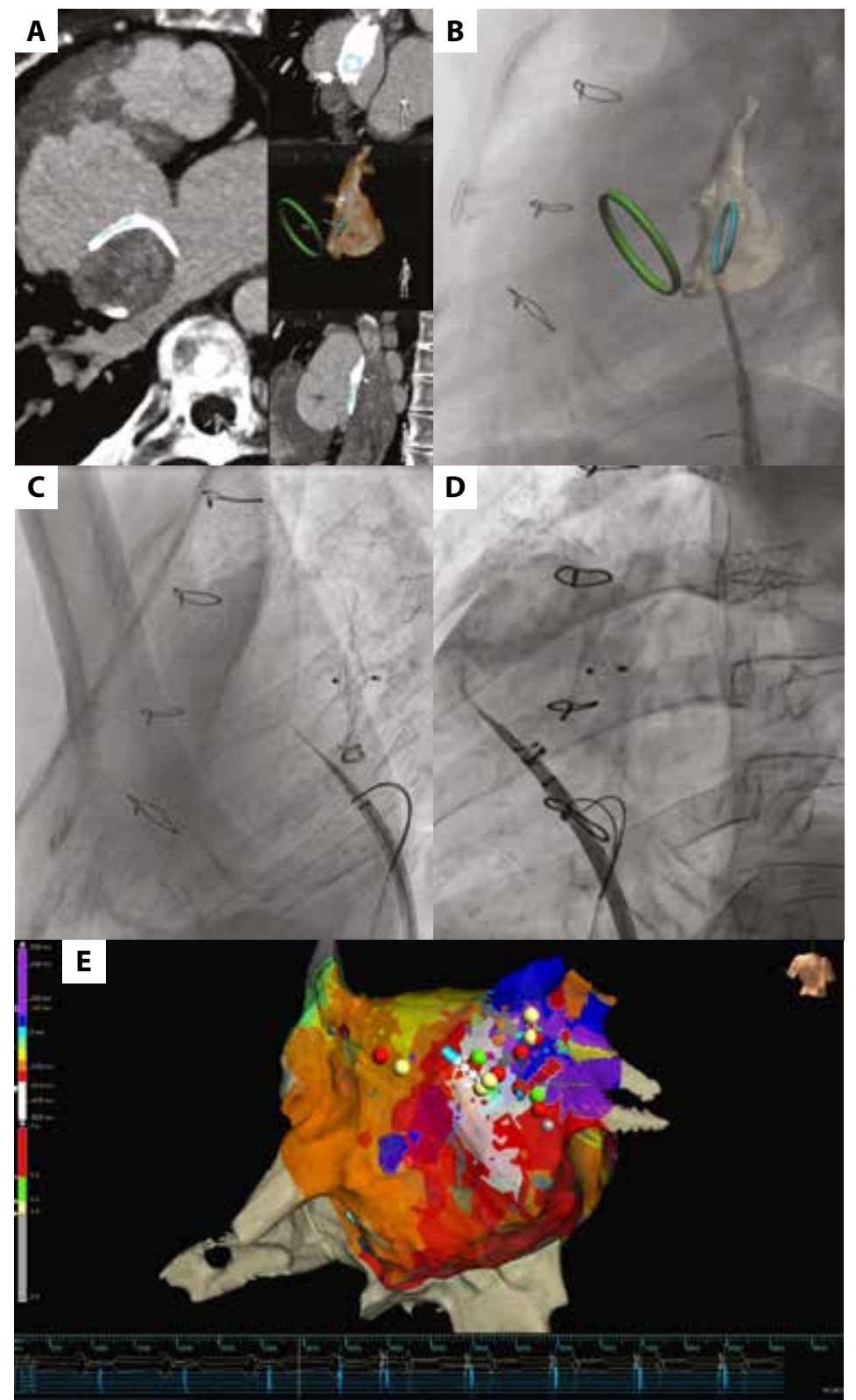

Figure 1. A. Preprocedural three-dimensional model of a septum between the Fontan tunnel and the right atrium preparation based on computed tomography cross-sectional scans. B. Image fusion with desired puncture location (blue ring) and tricuspid valve annulus (green ring) markings (left oblique projection) - the tip of the transseptal sheath at the bottom of the septum. C. The needle in the atrium after puncture. D. Control right atrium angiography through the dilator. E. Left atrium activation map - posterior wall; location of the ablation catheter in place of radiofrequency application larly useful in the treatment of patients with unique anatomy.

\section{Article information}

Conflict of interest: None declared.

Open access: This article is available in open access under Creative Common Attribution-Non-Commercial-No Derivatives 4.0 International (CC BY-NC-ND 4.0) license, allowing to download articles and share them with others as long as they credit the authors and the publisher, but without permission to change them in any way or use them commercially. For commercial use, please contact the journal office at kardiologiapolska@ ptkardio.pl.

How to cite: Gałeczka M, Kowalski O, Fiszer R. Fontan tunnel puncture with 3-dimensional image fusion guidance for ablation of supraventricular arrhythmia in a patient with unique anatomy. Kardiol Pol. 2021; 79(7-8): 873-874, doi: 10.33963/KP.15971.

\section{REFERENCES}

1. Rijnberg FM, Blom NA, SojakV, et al. A 45-year experience with the Fontan procedure: tachyarrhythmia, an important sign for adverse outcome. Interact Cardiovasc Thorac Surg. 2019; 29(3): 461-468, doi: 10.1093/icvts/ivz111, indexed in Pubmed: 31038168.

2. Bhaskaran A, Nayyar S, Charla P, et al. Lateral tunnel Fontan atrial tachycardia ablation trans-baffle access is not mandatory as the initial strategy. J Interv Card Electrophysiol. 2020; 58(3): 299-306, doi: 10.1007/s10840019-00580-5, indexed in Pubmed: 31399922.

3. LiD, Fan Q, Hirata Y, et al. Arrhythmias after Fontan operation with intra-atrial lateral tunnel versus extra-cardiac conduit: a systematic review and meta-analysis. Pediatr Cardiol. 2017; 38(4): 873-880, doi: 10.1007/s00246-0171595-8, indexed in Pubmed: 28271152.

4. Derejko P, Rybicka J, Biernacka EK, et al. Atrial tachycardia ablation in patients with a functional single ventricle after the Fontan surgery. Kardiol Pol.2016; 74(8):762-771, doi: 10.5603/KP.a2015.0214, indexed in Pubmed: 26575308.

5. Goreczny S, Dryzek P, Morgan GJ, et al. Novel three-dimensional image fusion software to facilitate guidance of complex cardiac catheterization: 3D image fusion for interventions in CHD. Pediatr Cardiol. 2017; 38(6): 1133-1142, doi: 10.1007/s00246-017-1627-4, indexed in Pubmed: 28551818. 